\title{
The Lived Experience of Myasthenia Gravis: A Patient-Led Analysis
}

\author{
Nancy Law $\cdot$ Kelly Davio $\cdot$ Melissa Blunck $\cdot$ Dawn Lobban · \\ Kenza Seddik
}

Received: June 10, 2021 / Accepted: September 16, 2021 / Published online: October 23, 2021

(c) The Author(s) 2021, corrected publication 2022

\section{ABSTRACT}

Introduction: A greater understanding of the reality of living with myasthenia gravis (MG) may improve management and outcomes for patients. However, there is little published data on the patient perspective of how MG impacts

Nancy Law and Kelly Davio contributed equally to this work.

Deceased: Nancy Law.

Supplementary Information The online version contains supplementary material available at https:// doi.org/10.1007/s40120-021-00285-w.

N. Law

Patient Author, MG Patient Advocate, Parker, CO, USA

K. Davio

Patient Author, MG Patient Advocate, Richmond, London, UK

M. Blunck

UCB Pharma, Smyrna, GA, USA

D. Lobban

Envision Pharma Group, Cheshire, UK

K. Seddik

UCB Pharma, Paris, France

K. Seddik $(\square)$

420 Rue d'Estienne d'Orves, 92700 Colombes, France

e-mail: Kenza.seddik@ucb.com life. Our objective was to reveal the lived experience of MG from the patient perspective.

Methods: This analysis was led by an international Patient Council comprising nine individuals living with $\mathrm{MG}$ who serve as local/national patient advocates in seven countries (Europe and the United States). Insights into the lived experience of MG were consolidated from three sources (a qualitative research study of 54 people with MG or their carers from seven countries; a previous Patient Council meeting [September 2019]; and a literature review). Insights were prioritised by the Patient Council, discussed during a virtual workshop (August 2020) and articulated in a series of statements organised into domains. Overarching themes that describe the lived experience of MG were identified by the patient authors.

Results: From 114 patient insights and supporting quotes, the Patient Council defined 44 summary statements organised into nine domains. Five overarching themes were identified that describe the lived experience of MG. These themes include living with fluctuating and unpredictable symptoms; a constant state of adaptation, continual assessment and tradeoffs in all aspects of life; treatment inertia, often resulting in under-treatment; a sense of disconnect with healthcare professionals; and feelings of anxiety, frustration, guilt, anger, loneliness and depression. 
Conclusion: This patient-driven analysis enriches our understanding of the reality of living with MG from the patient perspective.

Keywords: Myasthenia gravis; Lived experience; Patient perspective; Qualitative

\section{Key Summary Points}

\section{Why carry out this analysis?}

Few studies explore the impact of living with myasthenia gravis from the patient perspective.

A greater understanding of what it means to live with myasthenia gravis may improve patient care.

By collating and analysing patient insights, our aim was to describe the lived experience of myasthenia gravis from the patient perspective.

\section{What did we learn from this analysis?}

This international patient-led analysis of over 114 patient insights showed that living with myasthenia gravis significantly impacts many aspects of life.

Five themes that describe the experience of living with myasthenia gravis were articulated by the patient authors, including:

- living with fluctuating and unpredictable symptoms

- a constant state of adaptation, continual assessment and trade-offs in all aspects of life

- treatment inertia, often resulting in under-treatment

- a sense of disconnect with healthcare professionals

- feelings of anxiety, frustration, guilt, anger, loneliness and depression.

\section{INTRODUCTION}

Myasthenia gravis (MG) is a rare autoimmune disease characterised by antibody-mediated interference with neuromuscular transmission at the neuromuscular junction [1]. MG is classed as a rare disease, and its prevalence is estimated to be about 1-2 per 10,000 people [2]. However, reported incidence rates are increasing, partly due to improved diagnostic techniques and an increased awareness of the disease [3]. Despite a growing wealth of published literature, how much do we really understand about what it means to live with MG from the patient perspective?

MG manifests clinically as muscle weakness [4] and muscle fatiguability [5]. Although some patients (15\%) experience ocular symptoms only, the majority (85\%) have more generalised symptoms [4]. Indeed, an exacerbation of generalised muscle weakness can result in a lifethreatening myasthenic crisis, requiring respiratory support [6]. Beyond muscle weakness and muscle fatiguability, many patients also report central fatigue, experienced as a lack of physical and/or mental energy, which is associated with reduced quality of life [5]. Common comorbidities for MG include dyslipidemia, diabetes, hypertension and other autoimmune conditions such as thyroid disease $[7,8]$. Symptoms of these comorbidities, combined with side effects of their associated treatments, further add to the burden of disease experienced by people with MG.

Although the epidemiology and clinical presentation of MG are well documented, effective therapies that cure or prevent this disease remain elusive [4, 9]. Despite recent advances in the treatment of some other rare diseases [10], progress in the treatment of MG has not been rapid. Thymectomy is an effective option for patients with thymomas, and can also be considered for other patients with $\mathrm{MG}$ [11]. Current medications focus on managing symptoms (e.g., with acetylcholinesterase inhibitors) or providing non-specific chronic immunosuppression (e.g., corticosteroids, nonsteroidal immunosuppressants). Systemic biologics such as rituximab and eculizumab can be 
used to treat refractory disease. Plasma exchange and intravenous immunoglobulin are fast-acting treatments typically used in conjunction with intensive care to manage myasthenic crisis $[4,6]$. Ongoing clinical trials provide hope for additional treatment options in the near future [12].

Ongoing clinical research and interventional trials aim to better understand and address the physiological manifestations of MG, but they will tell us very little about what it is really like for patients to live with this rare disease. To achieve this, we need approaches that are informed by phenomenology, enabling us to explore MG from the perspective of those who have experienced it. Phenomenology encourages us to study an individual's lived experience of the world, providing new meanings and enhancing our own understanding [13, 14]. Despite ongoing debate regarding the best methodologies, there is growing acceptance of the importance of phenomenology in medicine, especially in understanding chronic illness [15]. Such approaches rely on detailed reports of first-person experiences, gained through open dialogue during which patients are asked about their own experiences. Indeed, the importance of understanding the patient perspective and their priorities, especially in rare diseases, is recognised by the regulators, with both the European Medicines Agency and the US Food and Drug Administration developing initiatives to ensure an active dialogue with patients $[16,17]$

By gaining a better understanding of the lived experience of $\mathrm{MG}$, we can bridge any gap between healthcare providers' (HCPs') perceptions of MG based on objective clinical data and the patients' perceptions of MG based on their subjective everyday experiences. An effective patient-physician relationship is particularly important for successfully managing a chronic rare disease and identifying shared treatment goals [18]. Not only will each individual patient have a unique lived experience, but their perspective of their own disease, and hence treatment goals, may also change over time [19]. Studies investigating patients' experiences of living with MG have reported on the impact of this condition on many aspects of life, ranging from simple daily activities, such as personal hygiene and chewing food, to larger parts of an individual's identity, such as employment and family life [20-23]. MG has also been shown to have a significant emotional and psychological impact on patients: there is a high prevalence of depression and anxiety in people living with MG [24, 25]. However, few studies have explored the first-person patient perspective to gain a detailed understanding of the multifaceted lived experience of MG [26].

The need for greater understanding of the reality of living with generalised MG was identified by a group of leading national patient advocates from around the world: the International Myasthenia Gravis Patient Council. This Patient Council, initially formed in 2018, provides advice and guidance to UCB Pharma to ensure a patient-led approach to improving treatment for people with MG. During a meeting in September 2019, the Patient Council, in collaboration with UCB Pharma, initiated a qualitative analysis of patient insights, collated from multiple sources, to create a detailed patient consensus of the lived experience of active, generalised MG. Here, we report the Patient Council analysis of collated insights and creation of detailed statements, spanning multiple aspects (domains) of life. As two authors of this article (NL and KD) are experienced patient advocates and members of the Patient Council, we went on to identify themes from the data that span domains and portray the lived experience of MG. These themes are discussed, along with some of the personal growth aspects of living with MG that were also identified from the insights. We believe that this is the first patient-led, patient-authored analysis of the lived experience of MG and hope that it will enhance understanding of this rare disease.

\section{METHODS}

This analysis, initiated and funded by UCB Pharma, was led by the Patient Council: a group of people living with MG who serve as leading patient advocates in their local communities across the globe, including Europe and the United States. They are a diverse group of adult 
men and women who are proficient in the English language. Participating members of the Patient Council are listed in the acknowledgements.

Having previously identified a need to better understand and report the lived experience of MG from the patient perspective, the Patient Council reconvened in August 2020 to review insights collated from multiple data sources. Their objective was to generate a series of statements that, on the basis of their collective experience of MG, they felt best represented the lived experience of generalised MG.

An overview of the process followed for insight identification, collation, prioritisation and analysis is shown in Fig. 1.

\section{Insight Identification and Extraction}

Insights were based on qualitative data collected directly from people with generalised $M G$ or reported in the literature. This approach was based on that previously reported to investigate the patient perspective in another rare disease [27]. A simple framework analysis was used by two researchers to systematically gather and categorise insights from three different sources:

1. A global qualitative research study of 54 people affected by MG (48 people with generalised MG and six caregivers; 39 female) from seven countries across Europe, the United States and Asia Pacific. Participants with MG were aged $\geq 18$ years, had experienced generalised symptoms for $\geq 1$ year (majority for $>5$ years) and were currently receiving treatment. Participants were interviewed between 1 November 2019 and 31 January 2020. Although the study itself investigated all aspects of the patient journey, only patient insights and quotes focused on the ongoing management of diagnosed patients receiving treatment for MG were extracted for this analysis.

2. A prior Patient Council meeting (Brussels, September 2019) report detailing discussions between six council members living

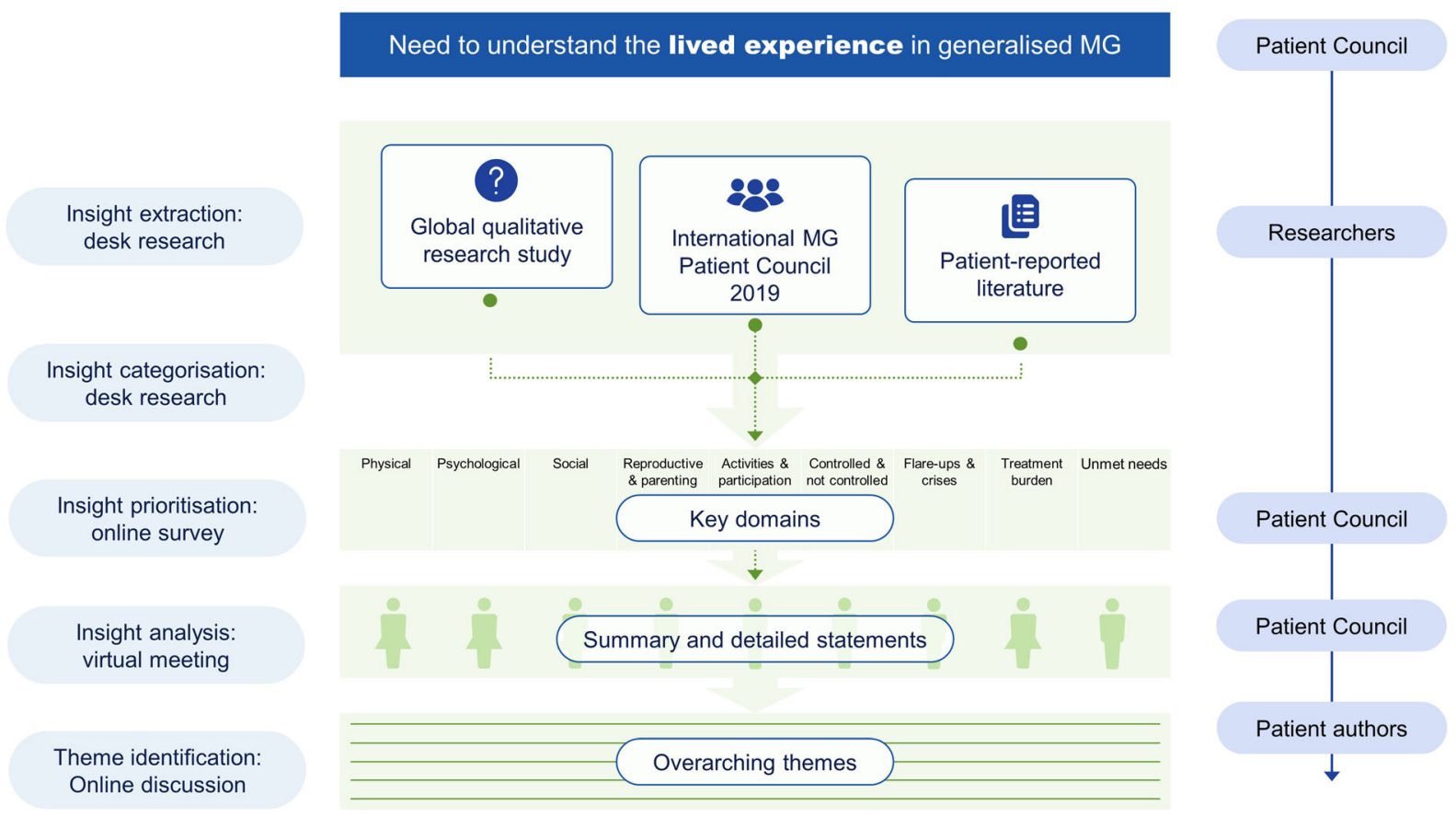

Fig. 1 The patient-led process followed for insight identification, collation, prioritisation and analysis. $M G$ myasthenia gravis 
with MG who serve as patient advocates in their local communities across the globe, including in Europe and the United States.

3. Selected articles (32 peer-reviewed research publications, one newsletter and one book) that present patient-reported outcomes or experiences of living with MG. The articles were identified by a comprehensive search of the literature (PubMed, predetermined patient and sociology journals, articles and books) and screened for relevance by two researchers.

Further details of these data sources are provided in the Electronic Supplementary Material.

With a lack of published data describing the lived experience of MG having been acknowledged at study initiation, data from the qualitative research study of people with MG were used as the primary source of insights. These insights were supplemented with any additional relevant insights from the prior Patient Council meeting (September 2019) output. Finally, any reported insights from the literature review not already captured from the two prior sources were included. Insights were only captured once, regardless of how many times they appeared in the data sources.

During extraction of the insights, care was taken to accurately represent the source data and minimise researcher interpretation: this included cross-checking and discussion between the two researchers. Specific patient quotes were also noted to further explain the insights. The insights and quotes were then categorised into descriptive domains reflecting different aspects of the lived experience of MG. Domains were initially constructed on the basis of suggestions proposed by the Patient Council at the start of the analysis, then modified slightly by the researchers during insight extraction to provide a framework with which to present the different aspects of the lived experience of MG.

\section{Insight Analysis by the Patient Council}

Collated insights and quotes, categorised by domain, were sent to the Patient Council members for review and prioritisation via an online survey. For each insight, the survey asked the Patient Council members to consider how well the insight represented the lived experience of MG from a patient perspective ('very well'/'somewhat'/'not very well'). For each domain, each Patient Council member was asked to choose the five insights that they felt best described the lived experience of MG for that domain. An open-ended comment box at the bottom of each domain asked the Patient Council members to 'Please add any supporting comments to explain your choices, anything that's missing, or anything you would like to add'. At the end of the survey, Patient Council members were asked 'Are there any positive aspects of living with $\mathrm{MG}$ as a chronic condition which you would like to share?'.

The findings of this survey were subsequently presented by the researchers and discussed with the Patient Council during a 7-h virtual meeting in August 2020. Detailed statements representing the lived experience of $M G$ for each domain were articulated, based on the insights. At this stage, insights could be reworded and/or merged to provide concise statements. Care was taken not to change the meaning of the insights. Quotes from people with MG considered by the Patient Council to be most descriptive of the lived experience for each domain were identified during the same online meeting.

Statements were finalised by the patient authors offline after the meeting. Each statement consisted of a succinct summary statement supported by more detailed statements to capture the nuances of the insights. Patient authors (NL and KD) subsequently met online (October 2020) to discuss the statements and draw out themes that were present across multiple domains and that they felt best described the lived experience of MG.

Neither the global qualitative study nor the Patient Council-led analysis was an investigation of clinical outcomes with any intervention. Therefore, neither ethics committee approval nor clinical trial registration was required. The patients who participated in the qualitative research study provided consent for their data to be published. Members of the Patient Council consented to the publication of their insights 
and analysis. All members of the 2020 Patient Council approved the statements.

\section{RESULTS}

\section{Insight Identification, Extraction and Analysis}

In total, 114 insights and 50 supporting quotes from people with MG were identified from across the three data sources and organised into nine domains: physical; psychological; social; reproduction and parenting; activities and participation; controlled and not controlled; flareups and myasthenic crises; treatment burden; and unmet needs. Definitions of the key domains are provided in Table 1.

\section{Summary Statements on the Lived Experience of MG}

The Patient Council defined a total of 44 summary statements describing the lived experience of MG across nine domains, and each domain was supported by a patient quote. Summary statements and a representative quote for each domain are shown in Table 1. The detailed statements can be found in Table S3.

\section{Key Themes Reflecting the Lived Experience}

Following this Patient Council-led exercise to create the statements, the patient authors (NL and $\mathrm{KD}$ ) reviewed the statements from across all domains and identified five overarching themes that described the lived experience of MG from the patient perspective. The detailed statements that best describe each of the themes are provided in Table 2 .

Theme 1: The challenge of living with MG extends beyond managing the characteristic muscle weakness. The fluctuating and unpredictable nature of symptoms, with periods of worsening and remission, has a substantial impact on the lives of people with MG.
'Every patient will have muscle weakness, but the difficulty to live with is that it is so unstable...the fluctuation is even worse to live with than the muscle weakness itself.' -Patient advocate, Patient Council.

Theme 2: As a consequence of living with fluctuating symptoms, people with MG navigate a constant state of adaptation to their muscle weakness. They have to make continuous assessments and trade-offs in all aspects of their life, including crucial areas such as work, family planning and treatment.

'You feel it from the moment you wake up and you have to adjust your routines and expectations; I live day by day. Those bad days you need to prioritise the most important activities, or the most basic, and try to work with your medication.'-Person with MG in the qualitative study.

Theme 3: Despite suboptimal disease control, there can be a reluctance among both patients and HCPs to alter their comfort zone of MG treatment. Multiple factors contribute to this 'treatment inertia', which can result in people with MG being under-treated [28, 29]. These include a lack of consensus on what constitutes optimal disease control, concerns over potential additional side effects and the time needed to see the benefits of a change in treatment. These factors can lead to a reluctance to 'rock the boat'. Furthermore, some patients who are not treated by a specialist can feel that their HCP does not fully understand their disease.

'Yes, if you don't know something is going to work, and it doesn't work, you feel like you've wasted 6 months which can be very frustrating.'-Patient advocate, Patient Council.

'We don't want to just survive-we want to thrive.'-Patient advocate, Patient Council.

Theme 4: People with MG may feel a sense of disconnect with their HCPs. This feeling is largely driven by barriers to communication such as limited time, a gap in the perception of both disease and treatment burden and differences in treatment goals. Although HCPs may focus on managing clinically relevant 
Table 1 Summary statements describing the lived experience of MG by key domain

Summary statements by domain

Most representative quote

\section{Physical (physical symptoms and impact of MG on the body)}

Muscle weakness and fatiguability are experienced by almost all people with MG

Muscle weakness in MG is unstable, fluctuating and unpredictable, which is difficult to live with and reduces quality of life

Generalised fatigue manifests as exhaustion in people with MG

People with MG can have disrupted sleep

The experience of MG differs between and within individuals

MG is not always progressive-it can improve

\section{Psychological (psychological impact of MG: intrapersonal}

MG is largely an 'invisible' illness, so other people may not understand its impact

The unpredictability and uncertainty of MG makes it hard for people to plan

Living with MG can lead to feelings of anger, frustration and depression

People with MG may have feelings of guilt

Living with MG can bring out optimism, hope and resilience

Emotional support is very important
'Having muscular weakness means that, on the worst days, holding up a hairdryer simply isn't possible, and that managing to get some mascara on without stabbing myself in the eyeball feels like a CV-worthy achievement. Putting on a dress with the zipper up the back, like in the dress in the ad? Not going to happen. Send in the leggings please'-Patient advocate, Patient Council

\section{[e.g., self] psychological and emotional needs)}

'You don't know how you will feel from one day to the next or what the future holds'-Person with MG in the qualitative study 
Table 1 continued

Summary statements by domain

Most representative quote

\section{Social (impact of MG on relationships with others: interpersonal needs)}

Living with MG means it can be a struggle to make plans, resulting in feelings of loneliness

MG can negatively impact relationships

People find comfort in connecting with other people with MG who understand
'Soon I couldn't breathe or speak well enough to chat with friends on the phone, much less meet for a social gathering. My world grew smaller and my close friends less numerous. I aimed my loneliness at books-both the reading and writing of them. But when my eyes couldn't work well enough to read a page, and when my muscles were too wobbly to allow me to write for more than a few moments at a time, I retreated even further in to the solitude of my mind'-Patient advocate, Patient Council

'A lot of people don't understand what MG means. They don't know what it is and they also don't want to learn about it. People would just rather withdraw from you. This really makes your social circle much, much smaller. I used to have a lot of friends and business partners as well, but all of this has fallen away. I only have a handful of very old friends that I have had since childhood. Everyone else is gone'-Person with MG in the qualitative study

Reproductive and parenting (impact of MG on reproductive and parenting choices-applicable to both women and men)

People with MG have concerns about pregnancy, both for themselves and for the baby

Concerns about the impact of MG and its treatment on their ability to cope as a mother can deter women from planning a pregnancy

Concerns about the impact of MG on the ability to cope as a father can deter men from planning for pregnancy with their partner

MG leads to 'trade-offs' for people with children

More education and guidance are needed regarding family planning for people with MG
'I was really willing to look after my children, but that made me getting worse. It was too difficult to fulfil a mother's responsibility with MG. I did not bring up my children by myself, I feel regret for losing intimacy with them. Meanwhile because of my disease, I was abandoned by my husband'-Patient quote from the literature review [21] 
Table 1 continued

Summary statements by domain

Most representative quote

\section{Activities and participation (impact of MG on activities of daily life)}

Activities can take longer and may need to be limited for people with $\mathrm{MG}$

Activities need more planning for people with $\mathrm{MG}$

MG can negatively impact employment and schooling

People with MG (perhaps younger people in particular) feel a sense of loss due to restrictions in activity and limitations in life choices
'You feel it from the moment you wake up and you have to adjust your routines and expectations, I live day by day. Those bad days you need to prioritize the most important activities, or the most basic, and try to work with your medication'-Person with MG in the qualitative study

Controlled and not controlled (includes the experience of living with both controlled and 'inadequately controlled' MG, and living with trade-offs)

MG could be better controlled in a significant proportion of 'It is particularly frustrating [for people with refractory people with the disease

There are differences in the level of control achieved for different people living with $\mathrm{MG}$

There is a level of inertia around switching treatments, even disease] to read that most people with MG are well managed on treatment, or have "normal" lives, when their experiences are quite the opposite'-Patient quote from the literature review [43] when they are not optimised

People with MG adapt to poorly or moderately controlled MG by developing coping strategies

Flare-ups or crises after a period of stability or remission are discouraging

Resilience helps people with MG cope

Flare-ups and myasthenic crises (impact of flare-ups and myasthenic crises-also includes people who are not responding to treatment)

People with MG fear a crisis

There are barriers to presenting early for medical help for a flare-up, including uncertainty about when and how best to do this

Concerns about potential flare-ups can make travel a challenge
'Whenever I think about joining in on strenuous activities with friends...I'm never sure how far I'll make it'-Person with MG in the qualitative study

'Turns out stress is my main trigger, and it's what caused those first symptoms to show up'-Person with MG in the qualitative study

\section{Treatment burden (related to treatment in general and specific to individual treatments)}

People with MG make trade-offs between treatment efficacy 'Living with the idea these medicines have bad side effects, and treatment burden

People with MG perceive a lack of communication of treatment burden from HCPs

Steroids have many adverse effects

Current treatments are very slow to take effect you want to get by on the lowest meds you can even if you know you could be stronger on $20 \mathrm{mg}$ instead of $5 \mathrm{mg}$, people are living with that trade off and that's why we need better treatments and not after everything else has failed you'-Patient advocate, Patient Council 
Table 1 continued

Summary statements by domain

Most representative quote

Unmet needs (unmet needs for people living with ongoing management of MG)

The ultimate goal is a cure for MG, but better long-term treatment efficacy and tolerability would be an improvement for patients

There is a need for improved treatment options for MG

People with MG want to hear about new treatments in development

Broad access to effective treatment for MG is critical

There is a need for better understanding from HCPs on the treatment goals of people with $\mathrm{MG}$

Technology can improve engagement and education in MG

Psychological support for people with MG could be improved

Detailed statements are provided in Table S3

$H C P$ healthcare provider, $M G$ myasthenia gravis

symptoms and side effects, this management may not address the impact that MG has on people's lives and the degree to which they must compensate in order to live with their symptoms.

'There is a disconnect sometimes. One of the leading doctors in $M G$ was quoted in a magazine saying $80 \%$ of his patients are in remission. Patients say "He may think I'm in remission, but I'm taking 20-30 mg of prednisone, I have all these side effects. It's not adequate control.'"-Patient advocate, Patient Council.

Theme 5: MG can lead to feelings of anxiety, frustration, guilt, anger, loneliness and depression. These feelings may be driven by multiple factors, from the burden of $\mathrm{MG}$ symptoms themselves to social isolation, loss of control and a lack of support.

'You can't forget you have it, I feel tied to MG.'-Patient advocate, Patient Council.

'I remember a time when I couldn't go out for dinner with friends after the theatre because I
'Achieving a sustained effect so patients don't have to take so much medication'-Person with MG in the qualitative study was so exhausted. I cried a lot that evening.'-

Patient advocate, Patient Council.

\section{Personal Growth Aspects of Living with MG}

Insights provided by five members of the Patient Council in response to the final question of the online survey identified four personal growth aspects of living with MG. These include greater resilience and empathy, increased self-awareness, a better perspective on life and an opportunity to help others. These personal growth aspects with representative quotes are provided in Table 3.

\section{DISCUSSION}

Generalised MG has a profound impact on many aspects of the lives of those with the disease. This analysis of insights gathered from multiple sources provides a detailed description of the lived experience of MG from the patient perspective. By collating 114 different insights 
Table 2 Statements that support the key themes from across multiple domains

\section{Summary statements that support themes}

Detailed statements that support themes

Theme 1: The challenge of living with MG extends beyond managing the characteristic muscle weakness. The fluctuating and unpredictable nature of symptoms, with periods of worsening and remission, has a substantial impact on the lives of people with $M G$

Muscle weakness in MG is unstable, fluctuating and unpredictable, which is difficult to live with and reduces quality of life

The experience of MG differs between and within individuals

Flare-ups or crises after a period of stability or remission are discouraging
The muscle weakness associated with MG is unstable and fluctuating in nature: the weakness comes and goes. The fluctuations are particularly difficult to live with and can have a significant, debilitating impact on people's lives owing to the unpredictability, which affects their ability to plan and diminishes all aspects of their life

MG is a variable and unpredictable condition, which means that the experience is different for each person, and also differs for each individual at different times ('good days' and 'bad days')

In addition, people with MG report that the importance of MG subtype (anti-MuSK and anti-AChR) for treatment decisions varies by country. There is an awareness amongst people with MG that anti-MuSK MG is more difficult to stabilise, leading to a greater physical toll compared with the anti-AChR subtype. People with MG may be categorised as 'seropositive' (testing positive for antibodies to anti-MuSK, anti-AChR or other relevant antibodies) or 'seronegative' (testing negative to one, more or all of the relevant antibodies)

When the condition is stable or in remission, people can begin to resume their pre-MG activities-and can even 'forget' they have MG. This can make flare-ups or crises even more discouraging and depressing, as they are forced to adapt all over again

Theme 2: As a consequence of living with fluctuating symptoms, people with $M G$ navigate a constant state of adaptation to their muscle weakness. They have to make continuous assessments and trade-offs in all aspects of their life, including crucial areas such as work, family planning and treatment

The unpredictability and uncertainty of MG makes it hard The unpredictability of MG impacts the ability of people for people to plan living with MG to plan, in both the short and longer term. The feeling of not having any control over MG, and not knowing how they will feel on any given day, can lead to feelings of uncertainty and anxiety about the future 
Table 2 continued

Summary statements that support themes

People with MG adapt to poorly or moderately controlled MG by developing coping strategies

Concerns about the impact of MG and its treatment on their ability to cope as a mother can deter women from planning a pregnancy

MG can negatively impact employment and schooling

\section{Detailed statements that support themes}

People with MG adapt to their new reality by implementing coping strategies to overcome MG-related restrictions when their condition is not fully under control. They may even 'over-adapt' and seem that they are coping well, when in fact they may be able to live with fewer adaptations if their treatment was optimised. They rely on these adaptations and coping mechanisms, such as long-term planning, allowing for more frequent breaks, changing or reducing the amount or type of work they do, proactively cancelling plans if necessary and adapting the ways in which they conduct activities of daily living such as eating or personal hygiene. The fluctuating nature of MG means there may be a need for constant 'mental calculus' involving calibration of activities and coping strategies

People with MG may consider abstaining from having children because of the condition. People with MG express concerns about adding additional stress on their relationships, or not having the physical strength to carry the baby or cope with its needs

Employment can present challenges for people with MG as they may not be able to manage a full-time role. Deskbased as well as manual jobs can be difficult; for example, people may have problems with eye fatigue, neck pain, ability to smile or speak, or staying alert. The impact of MG on their ability to drive can also be a barrier to employment and affect people's sense of independence. People with MG are also often concerned about the emotional stresses of MG manifesting themselves in the workplace, or, conversely, the stress of work negatively impacting their condition. Employer attitudes can vary significantly by country and workplace and can even affect the treatments people with MG are able to commit to; for example, they may not feel able to take time away from work to receive IVIg treatment. Many of these issues are also relevant to the ability of both children and adults with MG to pursue educational activities 
Table 2 continued

\section{Summary statements that support themes}

People with MG make trade-offs between treatment efficacy and burden

\section{Detailed statements that support themes}

Many people with MG are living with trade-offs between feeling better and having adverse effects from their treatments that diminish their quality of life. Some are willing to accept a greater treatment burden in exchange for greater efficacy. For example, although IVIg can be an effective treatment, some people experience intolerable adverse effects. There is also a significant time commitment to receive the intravenous treatment, and it may not be available in all locations There are concerns around IVIg shortages, particularly considering the decrease in plasma donations during the current COVID19 pandemic

Theme 3: Despite suboptimal disease control, there can be a reluctance among both patients and HCPs to alter their comfort zone of $M G$ treatment. Multiple factors contribute to this 'treatment inertia', which can result in people with $M G$ being undertreated [28, 29]. These include a lack of consensus on what constitutes optimal disease control, concerns over potential additional side effects and the time needed to see the benefits of a change in treatment. These factors can lead to a reluctance to 'rock the boat'. Furthermore, some patients who are not treated by a specialist can feel that their HCP does not fully understand their disease

There are differences in the level of control achieved for different people living with $M G$
People with 'well-controlled' MG are effectively treated with few or no residual symptoms. However, many people are living with inadequate levels of symptom control: People with 'moderately controlled' symptoms are adapting their lifestyle to compensate for suboptimal treatment; where this is not sufficient, people with 'poorly controlled' MG are experiencing symptoms that restrict their lives, despite their adaptations. The level of severity of 'poorly controlled' symptoms can be high (and dangerous), especially for those who are very good at compensating. People with MG that is not 'well controlled' are experiencing symptoms, in some cases severe, which could be due to either undertreatment or unresponsive disease 
Table 2 continued

Summary statements that support themes

There is a level of inertia around switching treatments, even when they are not optimised

People with MG fear a crisis

\section{Detailed statements that support themes}

There is a level of inertia, from both people with MG and HCPs, with respect to changing treatment. Some people live with 'poorly controlled' MG for extended periods; they may be under-treated but do not switch treatments. This can be driven by many different factors-for example, waiting to see if they improve; fear of the process and time to optimise a new treatment and/or of leaving an imperfect 'comfort zone' of the current medication; lack of access to new medications; and concerns over starting steroid treatment when the duration is unknown and there are long-term adverse effects

People with MG fear a crisis, especially if they have had previous traumatic experiences such as being intubated, which can leave people with PTSD-like psychological distress and a reluctance to return to hospital in future. The fear of inducing a crisis can leave both people with MG and their HCPs reluctant to 'rock the boat' by changing medication. This can also impact willingness to participate in clinical trials

Theme 4: People with MG may feel a sense of disconnect with their HCPs. This feeling is largely driven by barriers to communication such as limited time, a gap in the perception of both disease and treatment burden and differences in treatment goals. Although HCPs may focus on managing clinically relevant symptoms and side effects, this management may not address the impact that $M G$ has on people's lives and the degree to which they must compensate in order to live with their symptoms

There are barriers to presenting early for medical help for a When people with MG begin to experience a flare-up, it is flare-up difficult for them to know objectively when or how they should seek medical help, as this is not well defined. There is a feeling amongst some people with MG that HCPs, particularly those with less knowledge of the condition, do not understand or even believe MG symptoms, which can discourage people from seeking help. People with MG may wait to seek assistance, either to be sure it is a real flare-up, or because following previous bad healthcare experiences they may be reluctant to present with symptoms that they worry an HCP may consider to be 'too mild'. Some people don't like to 'bother' their HCPs, and for some the distance involved is a barrier to early presentation. In the current pandemic, fear of contracting COVID-19 in a healthcare facility is also a deterrent from seeking medical intervention 
Table 2 continued

\section{Summary statements that support themes}

People with MG perceive a lack of communication of treatment burden from HCPs

There is a need for better understanding from HCPs on the treatment goals of people with MG

More education and guidance are needed regarding family planning for people with MG

\section{Detailed statements that support themes}

Information from HCPs on treatment burden, in terms of adverse effects and monitoring, is typically lacking from the perspective of people with MG. Some HCPs may lack sufficient knowledge to clarify the risk-benefit balance of treatments. They may sometimes prioritise other comorbidities over MG, and there is a need for understanding and consideration of the impact of the treatments for these comorbidities on treatment for MG

There are differences in perception between people with MG and their HCPs in what is considered to be satisfactory symptom control and quality of life. There is a need for education for both patients and HCPs that increases communication about treatment goals, and the perception of what is successful treatment and satisfactory quality of life. The nature of questions an HCP asks people with MG can have a strong influence on the HCP's perception of the impact of the disease and thus how it is managed

People with MG need support with family planning, but express that this is largely unaddressed by HCPs. There is a need for more information and education for both HCPs and people with MG around MG and pregnancy and for better guidance on treatments during this time. Advice from HCPs can have a strong influence on people's decisions on whether or not to have children. This decision can, ultimately, have a consequential effect on relationships. There is an unmet need for effective treatments that allow women with MG to safely become pregnant 
Table 2 continued

Summary statements that support themes

Emotional support is very important

\section{Detailed statements that support themes}

Emotional support is very important for people with MG, whether through family relationships and friendships, faith-based groups, HCPs or other people living with MG

People with MG also find huge value in support groups, reporting that it is helpful to know 'they are not alone' and to hear about how others in a similar situation cope. Such groups provide a forum to help them express and validate their concerns about symptoms, can fill knowledge and support gaps left through disconnects with HCPs, can enrich their social life and provide rewarding opportunities to help others. Support groups can also enhance communication within existing relationships, as they help to increase understanding of MG amongst those closest to the people with MG, in particular if they are also able to attend together. Different ways to participate, with both face-to-face and technology-enabled options, are important to facilitate the involvement of the broadest possible range of people living with $\mathrm{MG}$

Theme 5: MG can lead to feelings of anxiety, frustration, guilt, anger, loneliness and depression. These feelings may be driven by multiple factors, from the burden of $M G$ symptoms themselves to social isolation, loss of control and a lack of support

MG is largely an 'invisible' illness, so other people may not Because MG is largely an 'invisible' illness that others cannot understand its impact see, people with MG feel that other people do not understand it or take it seriously, leading to feelings of isolation

MG can lead to feelings of anger, frustration and depression

People with MG experience anger and frustration, directed at both the condition and the burden of treatments, and sometimes toward other people who may offer unhelpful advice. In addition, people with MG may report feeling depressed

People with MG may have feelings of guilt

People with MG may have feelings of guilt, driven by different factors-for example, feeling they are letting family and friends down if they need to cancel plans, or wondering if they have done something to 'cause' MG

Living with MG means it can be a struggle to make plans, resulting in feelings of loneliness

The unpredictability of MG can make it hard for people to make plans in advance. Many learn to listen to their bodies and be honest with themselves. However, this often means cancelling plans at the last minute, needing to rely on others, and missing out on events with family and friends. People with MG can worry that others see them as unreliable, leaving them feeling frustrated and lonely 
Table 2 continued

\section{Summary statements that support themes}

Psychological support for people with MG could be improved

\section{Detailed statements that support themes}

The diagnosis of MG creates psychological as well as physical challenges. People with MG and their family members report an array of emotions, including depression, anger, anxiety and fear. Psychological support can help, but it can be difficult for people to find mental health professionals who understand the burden of chronic illness, in particular the nuances of the rare disease that is MG. Poor-quality psychological support, for example, from a therapist who does not have a deep understanding of MG, may be worse than no support

$A C h R$ acetylcholine receptor, $H C P$ healthcare provider, $I V I g$ intravenous immunoglobulin, $M G$ myasthenia gravis, $M u S K$ muscle-specific tyrosine kinase, PTSD post-traumatic stress disorder

Table 3 Personal growth aspects of living with MG

\begin{tabular}{|c|c|}
\hline Growth aspects & Related quotes from the patient advocates, Patient Council \\
\hline $\begin{array}{l}\text { MG can contribute to personal growth, as it builds } \\
\text { resilience and empathy }\end{array}$ & $\begin{array}{l}\text { 'Living with MG can build resilience and empathy. But so too can } \\
\text { living with any number of other conditions. I do not think there is a } \\
\text { special, MG-specific quality that can be developed as a result of } \\
\text { experiencing life as an MG patient' }\end{array}$ \\
\hline $\begin{array}{l}\text { MG can result in a feeling of knowing yourself } \\
\text { better: listening to your own body }\end{array}$ & 'What doesn't kill you makes you stronger!' \\
\hline $\begin{array}{l}\text { Living with } M G \text { puts other things in life into } \\
\text { perspective }\end{array}$ & $\begin{array}{l}\text { 'It helps you to figure out what really matters in your life and set } \\
\text { priorities. Things that might have caused you stress before you } \\
\text { nearly died in crisis become minor annoyances' }\end{array}$ \\
\hline $\begin{array}{l}\text { Living with MG can lead to opportunities to meet } \\
\text { and help people in the same position }\end{array}$ & $\begin{array}{l}\text { 'You have the opportunity to meet a lot of really amazing and } \\
\text { wonderful people that you might never have known, who will } \\
\text { enrich your life. It's great to be a part of a patient community at a } \\
\text { time when there is great hope for better treatments, and the } \\
\text { opportunity to engage in both advocacy and clinical trials' }\end{array}$ \\
\hline
\end{tabular}

$M G$ myasthenia gravis

describing nine different domains of life, we have created a series of 44 summary statements that represent what it can mean to live with generalised MG. Clearly, every person's experience is unique and varies over time, so these data do not accurately represent every patient with MG. However, by identifying themes that emerge from the data, supported by multiple detailed statements from across different life domains, we can begin to describe aspects of what it can mean to live with this rare disease from the patient perspective.

Although several studies have reported on the impact of muscle weakness itself [25], this 
being the clinical description of the disease, one of the strong emerging themes we identified from this analysis describes the experience of living with fluctuating weakness and an unpredictable body (Theme 1). As symptoms of MG can vary significantly from one day to the next, unpredictable fluctuations in muscle weakness and fatigue can be a key challenge that affects multiple areas of life, including physical, psychological and social aspects. Living with uncertainty can lead to feelings of vulnerability and can make planning extremely difficult. After a period of remission, during which people with MG may gain a sense of stability, an unexpected exacerbation of symptoms can be particularly discouraging, forcing people with MG to confront once again the limitations of their disease. This is consistent with data from a small phenomenological study in seven patients that identified overlapping themes of weakness, uncertainty and change in patients with MG [26], and reflects the experience of 'bodily uncertainty', which has been described in phenomenological studies of other neurological conditions [30]. Indeed, such fluctuating and unpredictable symptoms can lead to concern among patients that an assessment by their HCP during a specific consultation may not reflect their true experience of living with $\mathrm{MG}$ [31].

As a result of living with an unpredictable body, our analysis shows that people with MG report having to navigate a constant state of adaptation. This involves making continuous assessments and trade-offs in all aspects of their life, impacting both day-to-day and major life decisions (Theme 2). They develop and rely on coping strategies and long-term planning, while remaining flexible in order to adapt to the limitations of an unpredictable body. Consistent with published studies $[22,23,32]$, our statements show that tradeoffs can be significant, impacting important life choices such as family planning and career opportunities.

Indeed, our analysis indicates that some people with MG may appear to be coping well, but are actually making numerous conscious or unconscious adaptations to their lives that could be avoided if their treatment was optimised. Unfortunately, there is a level of inertia around modifying treatments for MG among both patients and their HCPs, which was evident from our statements and is captured in Theme 3. This is consistent with data from an electronic survey study in which patients reported satisfaction with their disease burden, despite being symptomatic [33]. These findings may in part be explained by a large, recent study of data from patient interviews and surveys that demonstrates the significant burden caused by the side effects of traditional MG treatments (including acetyl cholinesterase inhibitors and immunomodulating therapies) [34]. Another contributory factor may be the time it takes for some treatments to start working [35]. Indeed, our statements show that some people with MG may prefer to live with suboptimal control, choosing to make adaptations to their life rather than facing the uncertainty of changing medication. In some cases, the fear of making things worse, or causing a crisis, by changing treatment can result in people overcompensating and living with poorly controlled symptoms that can be dangerous.

In addition to treatment inertia, some people with MG report a sense of disconnect with their HCPs. Although HCPs tend to focus on managing clinically relevant symptoms and adverse events, this approach may not prioritise aspects of the condition that have the greatest impact on the lived experience. This disconnect in relation to treatment goals can result in some people with MG feeling that their HCPs don't understand what is important to them (Theme 4), further discouraging them from seeking medical help for poorly controlled symptoms.

Our final theme describes feelings of anxiety, frustration, guilt, anger, loneliness and depression among people with MG (Theme 5). The unpredictability of MG can make planning difficult, resulting in the repeated need to cancel arrangements. This in turn can lead to feelings of loneliness, defeat and guilt about being unreliable and having to let people down. Combined with anger and frustration with the burden of both the disease and treatment, and the sense of disconnect with their HCP, it is unsurprising that depression and anxiety are common in those living with MG [25]. Such 
feelings may also be exacerbated by the central fatigue experienced by people with MG $[36,37]$.

Despite facing many challenges as described in the statements, peoples' perspectives on life with a chronic disease such as MG may change over time. A large meta-synthesis of qualitative research reports describing patient experiences of living with a chronic illness presents a model of shifting perspectives about their disease that may not be linked to symptom exacerbation or remission, but may instead occur to enable patients to make sense of their lives [19]. Although there are no inherent clinical benefits of MG, some people do experience some sense of personal gain as a result of living with this disease, as has been reported for other chronic diseases [38] and illness-related trauma [39].

In particular, prioritised insights from our Patient Council support the view that living with MG, as with other chronic conditions, can provide an opportunity for personal growth: some patients report improved self-awareness and increased empathy. Gaining perspective was also reported, enabling patients to build resilience and reassess their priorities.

'It helps you to figure out what really matters in your life and set priorities. Things that might have caused you stress before you nearly died in crisis become minor annoyances.' -Patient advocate, Patient Council.

Indeed, coping strategies to encourage optimism, self-reliance and feelings of control have been shown to have a positive impact on the well-being of patients with MG [40]. In addition, the opportunity for people with MG to help others and the benefits associated with this were evident in our insight analysis and have been reported elsewhere [21, 40]. Providing peer support has been shown to improve morale and self-esteem, leading to a sense of empowerment [41].

Following a patient-led approach, we have collated and prioritised insights from people living with MG to provide a comprehensive overview of the lived experience of MG. By reflecting on their extensive experience as patient advocates for their communities, the Patient Council were able to identify insights that best represent the lived experience of MG from the patient perspective. Care was taken throughout the process of insight identification, collation and prioritisation to ensure that the final statements accurately reflect the original first-person data on which they were based. Nevertheless, we acknowledge that there are a number of limitations with this approach. Despite steps to limit researcher bias throughout insight identification and collation, including cross-checking of data and close collaboration between the two researchers, this will have inevitably influenced the initial list of insights presented to the Patient Council for prioritisation. Furthermore, although a strength of this study was the extent of data sources analysed and the number of insights extracted (114 in total across three data sources), there is a risk that some insights became decontextualised during extraction and may not be applicable to other settings [42]. Finally, although the Patient Council involved in this analysis consisted of advocates from seven different countries, both male and female and representing a wide age range, a council of different advocates with different experiences may have prioritised different insights, impacting the final statements.

\section{CONCLUSIONS}

Despite these limitations, we believe that this patient-led analysis provides an important portrayal of the lived experience of MG from the patient perspective. More studies that present the patient experience are needed to build on our initial findings. Such studies could explore insights from larger patient populations, as well as carers, family and close friends, from across the globe to further enrich our understanding of what it really means to live with generalised MG. We hope that this analysis and the resulting statements describing the lived experience of $\mathrm{MG}$ from the patient perspective will enhance HCPs' understanding of this rare disease, thereby facilitating dialogue with patients and encouraging shared decision-making. 


\section{ACKNOWLEDGEMENTS}

The authors and UCB gratefully acknowledge the valued contribution of the first author, Nancy Law, who sadly passed away following preparation of this manuscript (Thursday, 23 September 2021). Nancy was instrumental in driving this important analysis to ensure that the voices of people living with myasthenia gravis are clearly heard. Her passion for and dedication to improving the lives of people living with myasthenia gravis will continue to be an inspiration to us all. We give special thanks to the people with MG who participated in the qualitative research survey. We thank the members of the International Myasthenia Gravis Patient Council: Patient Council September 2019: Johan Voerman (Netherlands); Claudia Schlemminger (Germany); Nancy Law (USA); Eva Frostell (Finland); Marguerite Friconneau (France); Annie Archer (France); Laura Maria Julia Calvo (Spain); Kelly Davio (UK). Patient Council August 2020 and pre-meeting survey: Annie Archer (France); Kelly Davio (UK); Eva Frostell (Finland); Hilde Kerkhofs (Belgium); Nancy Law (USA); Louis Loontjens (Belgium); Matthieu Luisgnan (France); Raquel Pardo Gomez (Spain); Johan Voerman (the Netherlands).

Funding. This study was funded by UCB Pharma (Brussels, Belgium). The journal's rapid service fee was also funded by UCB Pharma (Brussels, Belgium).

Medical Writing, Editorial and Other Assistance. Branding Science Ltd. conducted the qualitative research survey, funded by UCB Pharma. Jenny Hepburn and Dawn Lobban, of Envision Pharma Group, performed the literature search, analysed the primary data sources, compiled the insights and facilitated the Patient Council survey and discussion, funded by UCB Pharma. Medical writing assistance in the preparation of this article was provided by Dawn Lobban, PhD, of Envision Pharma Group, and was funded by UCB Pharma. Veronica Porkess, PhD, of UCB Pharma, also provided publication and editorial support.
Authorship. All named authors meet the International Committee of Medical Journal Editors (ICMJE) criteria for authorship for this article, take responsibility for the integrity of the work as a whole and have given their approval for this version to be published.

Author Contributions. Dawn Lobban performed the role of researcher in this analysis. All authors were involved in the study design, interpretation and preparation of the manuscript. All authors approved the final manuscript. As members of the Patient Council, Nancy Law and Kelly Davio were also involved in the insight selection and discussion. No funding was provided for authorship.

Prior Presentation. This manuscript is based on work that has been presented as a poster presentation at The American Association of Neurology Annual Meeting, 2021. Law N, Davio K, Blunck, M, et al. The lived experience of myasthenia gravis: A patient-led analysis. Poster presentation at the AAN Virtual Annual Meeting; 17-22 April 2021.

Disclosures. Nancy Law and Kelly Davio are members of the Patient Council. Melissa Blunck and Kenza Seddik are employees of UCB Pharma, which funded the study. Dawn Lobban is an employee of Envision Pharma Group, which was funded by UCB Pharma to support with the analysis and medical writing support.

Compliance with Ethics Guidelines. Neither the global qualitative study nor the Patient Council-led analysis was an investigation of clinical outcomes with any intervention. The global qualitative study adhered to local market research guidelines in the countries included and was conducted in accordance with EphMRA Code of Conduct. Therefore, neither ethics committee approval nor clinical trial registration was required. The patients who participated in the qualitative research study were fully informed of the purpose of research, provided consent to participate and agreed for their data to be published. Members of the Patient Council consented to the publication of their insights and analysis. 
Data Availability. All data generated or analysed during this study are included as supplementary information files.

Open Access. This article is licensed under a Creative Commons Attribution-NonCommercial 4.0 International License, which permits any non-commercial use, sharing, adaptation, distribution and reproduction in any medium or format, as long as you give appropriate credit to the original author(s) and the source, provide a link to the Creative Commons licence, and indicate if changes were made. The images or other third party material in this article are included in the article's Creative Commons licence, unless indicated otherwise in a credit line to the material. If material is not included in the article's Creative Commons licence and your intended use is not permitted by statutory regulation or exceeds the permitted use, you will need to obtain permission directly from the copyright holder. To view a copy of this licence, visit http:// creativecommons.org/licenses/by-nc/4.0/.

\section{REFERENCES}

1. Gilhus NE, Verschuuren JJ. Myasthenia gravis: subgroup classification and therapeutic strategies. Lancet Neurol. 2015;14:1023-36. https://doi.org/ 10.1016/s1474-4422(15)00145-3.

2. Carr AS, Cardwell CR, McCarron PO, McConville J. A systematic review of population based epidemiological studies in myasthenia gravis. BMC Neurol. 2010;10:46. https://doi.org/10.1186/1471-2377-1046.

3. Westerberg E, Punga AR. Epidemiology of myasthenia gravis in Sweden 2006-2016. Brain Behav. 2020;10: e01819. https://doi.org/10.1002/brb3. 1819.

4. Gilhus NE, Tzartos S, Evoli A, Palace J, Burns TM, Verschuuren J. Myasthenia gravis. Nat Rev Dis Primers. 2019;5:30. https://doi.org/10.1038/s41572019-0079-y.

5. Ruiter AM, Verschuuren J, Tannemaat MR. Fatigue in patients with myasthenia gravis. A systematic review of the literature. Neuromuscul Disord. 2020;30:631-9. https://doi.org/10.1016/j.nmd.
6. Hogan C, Lee J, Sleigh BC, Banerjee PR, Ganti L. Acute myasthenia crisis: a critical emergency department differential. Cureus. 2020;12: e9760. https://doi.org/10.7759/cureus.9760.

7. Diaz BC, Flores-Gavilán P, García-Ramos G, Lorenzana-Mendoza NA. Myasthenia gravis and its comorbidities. J Neurol Neurophysiol. 2015. https://doi.org/10.4172/2155-9562.1000317.

8. Tanovska N, Novotni G, Sazdova-Burneska S, et al. Myasthenia gravis and associated diseases. Open Access Maced J Med Sci. 2018;6:472-8. https://doi. org/10.3889/oamjms.2018.110.

9. Sanders DB, Wolfe GI, Benatar M, et al. International consensus guidance for management of myasthenia gravis: executive summary. Neurology. 2016;87:419-25. https://doi.org/10.1212/wnl. 0000000000002790 .

10. Kaufmann P, Pariser AR, Austin C. From scientific discovery to treatments for rare diseases-the view from the National Center for Advancing Translational Sciences-Office of Rare Diseases Research. Orphanet J Rare Dis. 2018;13:196. https://doi.org/ 10.1186/s13023-018-0936-x.

11. Aydin Y, Ulas AB, Mutlu V, Colak A, Eroglu A. Thymectomy in myasthenia gravis. Eurasian J Med. 2017;49:48-52. eurasianjmed.2017.17009.

12. Lascano AM, Lalive PH. Update in immunosuppressive therapy of myasthenia gravis. Autoimmun Rev. 2021;20: 102712. https://doi.org/10.1016/j. autrev.2020.102712.

13. Neubauer BE, Witkop CT, Varpio L. How phenomenology can help us learn from the experiences of others. Perspect Med Educ. 2019;8:90-7. https:// doi.org/10.1007/s40037-019-0509-2.

14. Carel H. Phenomenology and its application in medicine. Theor Med Bioeth. 2011;32:33-46. https://doi.org/10.1007/s11017-010-9161-x.

15. Svenaeus F. A defense of the phenomenological account of health and illness. J Med Philos. 2019;44:459-78. https://doi.org/10.1093/jmp/ jhz013.

16. European Medicines Agency. Patients and consumers. https://www.ema.europa.eu/en/partnersnetworks/patients-consumers. Accessed 17 Feb 2021.

17. US Food \& Drug Administration. Patient listening sessions. 2020. https://www.fda.gov/patients/learnabout-fda-patient-engagement/patient-listeningsessions. Accessed 17 Feb 2021. 
18. Garrino L, Picco E, Finiguerra I, Rossi D, Simone P, Roccatello D. Living with and treating rare diseases: experiences of patients and professional health care providers. Qual Health Res. 2015;25:636-51. https://doi.org/10.1177/1049732315570116.

19. Paterson BL. The shifting perspectives model of chronic illness. J Nurs Scholarsh. 2001;33:21-6. https://doi.org/10.1111/j.1547-5069.2001.00021.x.

20. Cioncoloni D, Casali S, Ginanneschi F, et al. Major motor-functional determinants associated with poor self-reported health-related quality of life in myasthenia gravis patients. Neurol Sci. 2016;37: 717-23. https://doi.org/10.1007/s10072-016-25563.

21. Chen YT, Shih FJ, Hayter M, Hou CC, Yeh JH. Experiences of living with myasthenia gravis: a qualitative study with Taiwanese people. J Neurosci Nurs. 2013;45:E3-10. https://doi.org/10.1097/JNN. Ob013e31828291a6.

22. Nagane Y, Murai H, Imai T, et al. Social disadvantages associated with myasthenia gravis and its treatment: a multicentre cross-sectional study. BMJ Open. 2017;7: e013278. https://doi.org/10.1136/ bmjopen-2016-013278.

23. Frost A, Svendsen ML, Rahbek J, Stapelfeldt CM, Nielsen CV, Lund T. Labour market participation and sick leave among patients diagnosed with myasthenia gravis in Denmark 1997-2011: a Danish nationwide cohort study. BMC Neurol. 2016;16: 224. https://doi.org/10.1186/s12883-016-0757-2.

24. Stewart SB, Robertson KR, Johnson KM, Howard JF Jr. The prevalence of depression in myasthenia gravis. J Clin Neuromuscul Dis. 2007;8:111-5. https://doi.org/10.1097/CND.0b013e3180316324.

25. Braz NFT, Rocha NP, Vieira ÉLM, et al. Muscle strength and psychiatric symptoms influence health-related quality of life in patients with myasthenia gravis. J Clin Neurosci. 2018;50:41-4. https://doi.org/10.1016/j.jocn.2018.01.011.

26. Keer-Keer T. The lived experience of adults with myasthenia gravis: a phenomenological study. AJON. 2015;25:40-6. https://doi.org/10.21307/ ajon-2017-112.

27. Mathias SD, Gao SK, Miller KL, et al. Impact of chronic immune thrombocytopenic purpura (ITP) on health-related quality of life: a conceptual model starting with the patient perspective. Health Qual Life Outcomes. 2008;6:13. https://doi.org/10. 1186/1477-7525-6-13.

28. American Diabetes Association. Therapeutic inertia-a brief discussion and definition. https:// professional.diabetes.org/meetings/definingtherapeutic-inertia. Accessed 24 Mar 2021.

29. Cutter G, Xin H, Aban I, et al. Cross-sectional analysis of the Myasthenia Gravis Patient Registry: disability and treatment. Muscle Nerve. 2019;60: 707-15. https://doi.org/10.1002/mus.26695.

30. van der Meide H, Teunissen T, Collard P, Visse M, Visser LH. The mindful body: a phenomenology of the body with multiple sclerosis. Qual Health Res. 2018;28:2239-49. 1049732318796831 . https://doi.org/10.1177/

31. Barnett C, Bril V, Kapral M, Kulkarni A, Davis AM. A conceptual framework for evaluating impairments in myasthenia gravis. PLoS ONE. 2014;9: e98089. https://doi.org/10.1371/journal.pone.0098089.

32. Ohlraun S, Hoffmann S, Klehmet J, et al. Impact of myasthenia gravis on family planning: how do women with myasthenia gravis decide and why? Muscle Nerve. 2015;52:371-9. https://doi.org/10. $1002 /$ mus. 24556 .

33. Mendoza M, Tran C, Bril V, Katzberg HD, Barnett C. Patient-acceptable symptom states in myasthenia gravis. Neurology. 2020;95:e1617-28. https://doi. org/10.1212/wnl.0000000000010574.

34. Bacci ED, Coyne KS, Poon JL, Harris L, Boscoe AN. Understanding side effects of therapy for myasthenia gravis and their impact on daily life. BMC Neurol. 2019;19:335. https://doi.org/10.1186/ s12883-019-1573-2.

35. Gotterer L, Li Y. Maintenance immunosuppression in myasthenia gravis. J Neurol Sci. 2016;369: 294-302. https://doi.org/10.1016/j.jns.2016.08. 057.

36. Tran C, Bril V, Katzberg HD, Barnett C. Fatigue is a relevant outcome in patients with myasthenia gravis. Muscle Nerve. 2018;58:197-203. https://doi. org/10.1002/mus.26069.

37. Hoffmann S, Ramm J, Grittner U, Kohler S, Siedler J, Meisel A. Fatigue in myasthenia gravis: risk factors and impact on quality of life. Brain Behav. 2016;6: e00538. https://doi.org/10.1002/brb3.538.

38. Paterson B, Thorne S, Crawford J, Tarko M. Living with diabetes as a transformational experience. Qual Health Res. 1999;9:786-802. https://doi.org/ $10.1177 / 104973299129122289$.

39. Hefferon K, Grealy M, Mutrie N. Post-traumatic growth and life threatening physical illness: a systematic review of the qualitative literature. $\mathrm{Br} \mathrm{J}$ Health Psychol. 2009;14:343-78. https://doi.org/ $10.1348 / 135910708 \times 332936$. 
40. Koopman WJ, LeBlanc N, Fowler S, Nicolle MW, Hulley D. Hope, coping, and quality of life in adults with myasthenia gravis. Can J Neurosci Nurs. 2016;38:56-64.

41. Embuldeniya G, Veinot P, Bell E, et al. The experience and impact of chronic disease peer support interventions: a qualitative synthesis. Patient Educ Couns. 2013;92:3-12. https://doi.org/10.1016/j. pec.2013.02.002.
42. Thomas J, Harden A. Methods for the thematic synthesis of qualitative research in systematic reviews. BMC Med Res Methodol. 2008;8:45. https://doi.org/10.1186/1471-2288-8-45.

43. Schneider-Gold C, Hagenacker T, Melzer N, Ruck T. Understanding the burden of refractory myasthenia gravis. Ther Adv Neurol Disord. 2019;12: 1756286419832242. https://doi.org/10.1177/ 1756286419832242. 